\title{
Pre-operative biomarkers and imaging tests as predictors of post-operative delirium in non-cardiac surgical patients: a systematic review
}

\author{
Farrah Ayob ${ }^{1}$, Enoch Lam ${ }^{1}$, George Ho ${ }^{2}$, Frances Chung ${ }^{1}$, Hossam El-Beheiry ${ }^{3}$ and Jean Wong ${ }^{1,4^{*}}$ (D)
}

\begin{abstract}
Background: Post-operative delirium (POD) is a common post-operative complication in elderly individuals and imposes a significant health and financial burden. Identifying predictive biomarkers may help understand the pathophysiology of POD. Our objective is to summarize the evidence of pre-operative biomarkers and imaging tests to predict POD in patients undergoing non-cardiac surgery.

Methods: A systematic search of English language articles in MEDLINE, EMBASE, Cochrane Database, PsychINFO, PubMed and ClinicalTrials. Gov up to January 2018 was performed. Studies that used biomarkers or imaging tests to predict POD and a validated POD assessment tool were included. Animal studies, paediatric, cardiac and intracranial surgery were excluded. Risk of bias was assessed using the Quality In Prognosis Study tool.

Results: Thirty-four prospective cohort studies involving 4424 patients were included. Nineteen studies described serum tests [Interleukin-6, Insulin-like Growth Factor 1, C-Reactive Protein (CRP), cholinesterases, apolipoprotein-E genotype, leptin, hypovitaminosis, hypoalbuminaemia, gamma-amino butyric acid], 10 described cerebral-spinal fluid tests (monoamine precursor, melatonin, acute phase proteins, S100B and neurofibrillary tangles), and 5 described imaging tests. Two studies had high risk of bias due to unclear outcome measurement and study participation. CRP was significantly associated with POD in 5 studies. Other biomarkers were either examined by only a single study or two or more studies with conflicting results.
\end{abstract}

Conclusion: CRP is the most promising biomarker associated with POD. However, we are still in the early stages in identifying biomarkers and imaging tests that may further understanding of the pathophysiology of POD.

Keywords: Systematic review, Predictive biomarkers and imaging, Predictive tests, Post-operative delirium, Predicting post-operative delirium

\section{Background}

Delirium is a common problem in hospitalized elderly individuals; it is defined as an acute onset of impaired cognitive functions, disturbance in attention and awareness that is not due to a pre-existing, established, or evolving neurocognitive disorder. Post-operative delirium (POD) usually

\footnotetext{
* Correspondence: jean.wong@unh.ca

'Department of Anesthesia, Toronto Western Hospital, University Health Network, 2-434 McLaughlin Wing, 399 Bathurst Street, Toronto, ON M5T 258, Canada

${ }^{4}$ Women's College Hospital, Toronto, Ontario, 76 Grenville St, Toronto, ON M5S 1B2, Canada

Full list of author information is available at the end of the article
}

emerges on post-operative day 1 to 3 , after a lucid interval after emergence from anaesthesia [1]. The distinction between emergence agitation, post- anaesthesia care unit (PACU) delirium and POD are difficult to distinguish, their definitions have been based on review of literature and expert opinion [2]. Emergence agitation has been defined as agitation after discontinuation of inhaled anaesthetics, while PACU delirium has been defined as positive delirium signs after 30 min stay in PACU until discharge from PACU (delirium signs upon arrival in PACU may be considered continuation of emergence from general anaesthesia) [2]. PACU delirium may be a subset of POD

(c) The Author(s). 2019 Open Access This article is distributed under the terms of the Creative Commons Attribution 4.0 International License (http://creativecommons.org/licenses/by/4.0/), which permits unrestricted use, distribution, and 
and patients who experience PACU delirium are reported to have higher risk of POD [3].

The aetiology of POD is poorly understood but is probably due to the presence of underlying mechanisms of immune activation, oxidative stress and neurotransmitter imbalances in predisposed individuals [4]. Serious short and long-term adverse outcomes include persistent functional decline, prolonged hospital stay, higher morbidity and mortality, and increased health care costs.

Many delirium risk prediction models have low specificity [5]. There is increasing interest in investigating the pathogenesis of POD as reflected by the high number of studies examining various biomarkers. Investigating possible biomarkers to identify high-risk patients may advance understanding about the pathophysiology of delirium. This systematic review aims to summarize the current evidence with respect to biomarkers and imaging tests that may predict POD in non-cardiac surgery patients.

\section{Methods}

\section{Search strategy}

A systematic search of the literature was performed according to Preferred Reporting Items for Systematic Reviews and Meta-Analyses statement (PRISMA) guidelines, Fig. 1. A comprehensive electronic search with the assistance of an expert medical librarian was conducted. The following databases were searched; MEDLINE (1946 to January 2018), Medline In-Process (up to January 2018), EMBASE (1947 to January 2018), Cochrane Central Register of Controlled Trials (up to December 2017), Cochrane Database of Systematic Reviews (2005 to January 2018), PsychINFO (1806 to January 2018), CINAHL, PubMed (up to January 2018) and ClinicalTrials. Gov (up to January 2018). We included randomized and non-randomized controlled trials, prospective and retrospective observational studies, and cross-sectional studies.

The search included a combination of the following MESH keywords "emergence delirium", "cognitive disorders", "cognition", "acute confusion", "delirium", "hallucination", "dementia", "mental", "post-anaesthesia", "symptom assessment", "algorithm surveys and questionnaires", "assess", "audit", "checklist", "focus group", "patient reported outcome measures", "interview", "instrument", "QoL", "screening", "survey", "pre-operative care", "pre-procedure", "post-operative", "evaluation", "validation", "DSM" and "Diagnostic and Statistical Manual of Mental disorders". The detailed search strategy is provided in Additional file 1.

\section{Study selection and data extraction}

Potentially eligible studies were reviewed independently by two reviewers (EL and GH). Title review and abstracts were screened to determine if the inclusion/exclusion criteria were fulfilled. Any disagreements or queries were resolved by consulting the senior author
(JW). Full text review of all included studies was conducted independently by the 3 reviewers (EL, GH and FA). Studies reporting the following were included: 1) Pre-operative biomarker testing for predicting POD, 2) imaging techniques to predict POD and 3) POD diagnosed by physician's assessment or a validated tool. Exclusion criteria were paediatric studies, animal studies, non-English publications, intracranial or cardiac surgery, abstracts and studies that assessed emergence agitation as POD in their analysis or used non-validated tools for delirium assessment. We also excluded studies that examined post-operative biomarkers. Information extracted from each study were: first author and year of publication, sample size, mean age for POD and non-POD groups, country, type of surgery, elective or emergent, pre-operative dementia, pre-operative investigation for delirium, method of diagnosis for POD and day of POD diagnosis.

\section{Assessment of methodological quality}

Each study was evaluated for risk of bias in 6 study domains using the Quality in Prognostic Studies (QUIPS) tool, (Additional file 2: Table S1) [6]. The QUIPS tool has been used widely for methodological quality in systematic reviews of risk prediction models. It assesses bias in prognostic factor studies; and rates 6 bias domains: 1) study participation (definition and description of the study subjects); 2) study attrition (study loss to follow-up); 3) prognostic factor measurement (quantitatively measured predictors of interest); 4) confounding measurement and handling; 5) outcome measurement (quantitatively measured outcome of interest) and; 6) statistical analysis (calibration and discrimination) as having high, moderate or low risk of bias [4]. Two reviewers (EL and GH) evaluated each study independently and discrepancies were resolved by analysing the weight of factors of bias described in the studies by a third author (FA).

\section{Results}

The initial electronic database search retrieved 6214 potential studies with 3 additional studies retrieved from citations (Fig. 1). After removal of 2679 duplicates and reviewing the titles and abstracts, 34 studies were included for final full text review. Since the predictive tests and the manner of results reporting was heterogeneous [e.g. odds ratio (OR) vs. $P$-values only], and the number of studies for each specific test was low, we did not perform a quantitative analysis and limited our review to qualitative evaluation.

\section{Description of included studies and indices}

POD assessment was performed using the Delirium Observation Screening Scale (DOSS) [7] Confusion Assessment Method (CAM) [8-36] or CAM-ICU [13, 24, 36], Delirium Rating Scale (DRS) [9, 15, 31, 37], Diagnostic and 


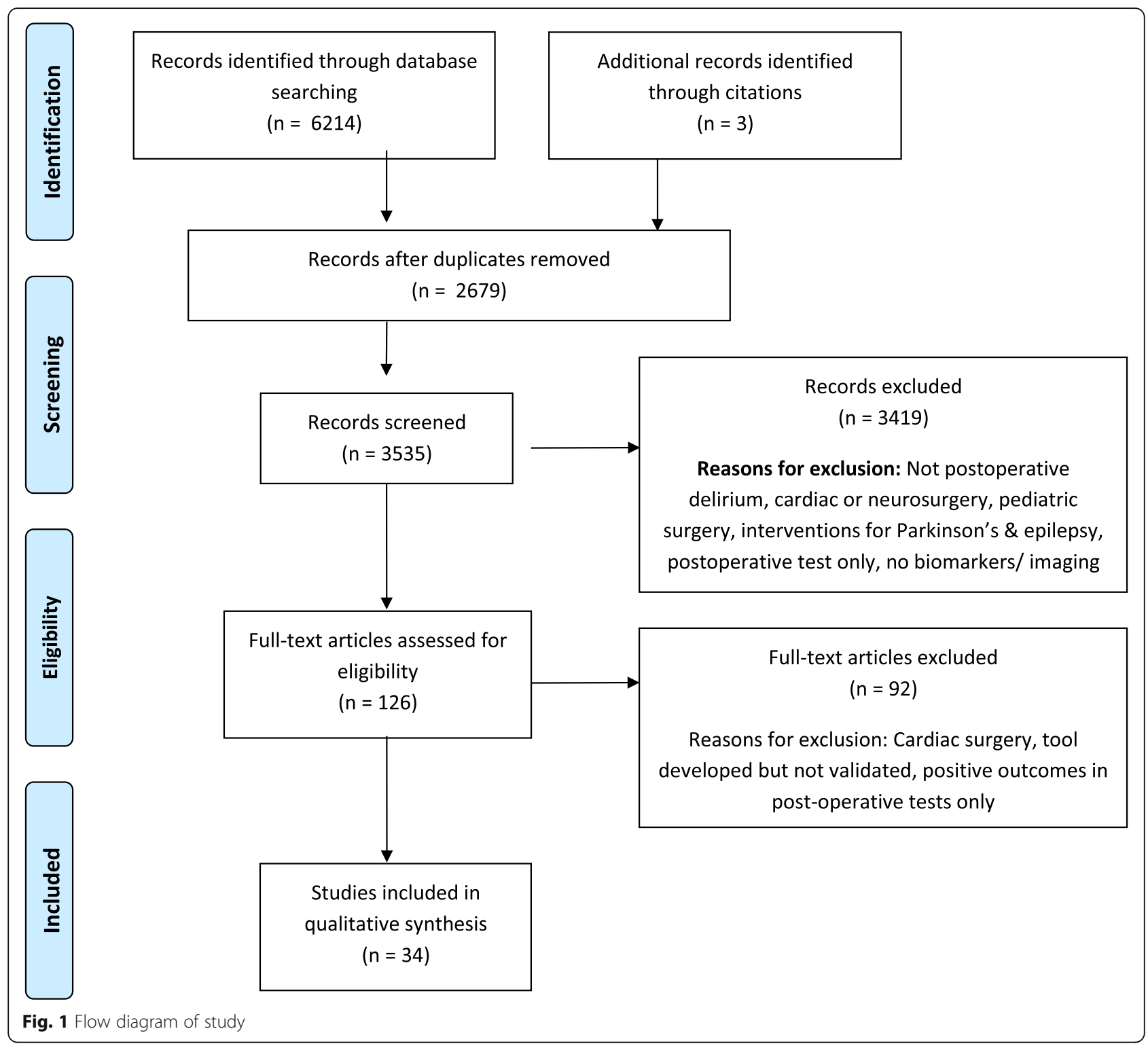

Statistical Manual of Mental Disorders (IV) [DSM IV] [7, 14, 36-39], or validated chart review [10, 21, 24, 34, 35]. Most studies assessed POD from the day of surgery, three studies assessed POD from post-operative day 2 onwards $[8,9,15]$, and 6 studies did not specify the time of POD assessment [7, 23, 35-37, 40]. In studies which assessed delirium on the day of surgery, none mentioned whether they included PACU delirium or distinguished between emergence agitation or POD.

\section{Study characteristics}

The main characteristics of the studies are shown in Table 1; serum biomarkers and Table 2; imaging tests. All were prospective cohort studies apart from one randomised controlled trial [41]. The 34 studies included data from
4424 patients. Nineteen studies described pre-operative serum biomarker tests $[8,10,12-25,37,38,41], 10$ described CSF biomarker tests [7, 9, 11, 26-33] and 5 described imaging tests [34-36, 40, 41]. Four studies investigated several different biomarkers $[9,11,14,17]$.

The studies were conducted in the United States, Europe and Asia. During data analysis, it was noted that several studies used the same patient population but examined different biomarkers. Specifically, 2 studies by Carejeira and colleagues [16, 17], and 6 studies used a subset from the Successful Aging after Elective Surgery (SAGE) study [10, 12, 21, 34-36]. The SAGE study was a prospective observational study evaluating the risk factors and long-term outcomes of delirium in 566 patients older than 70 years undergoing major non-cardiac surgery [39]. 


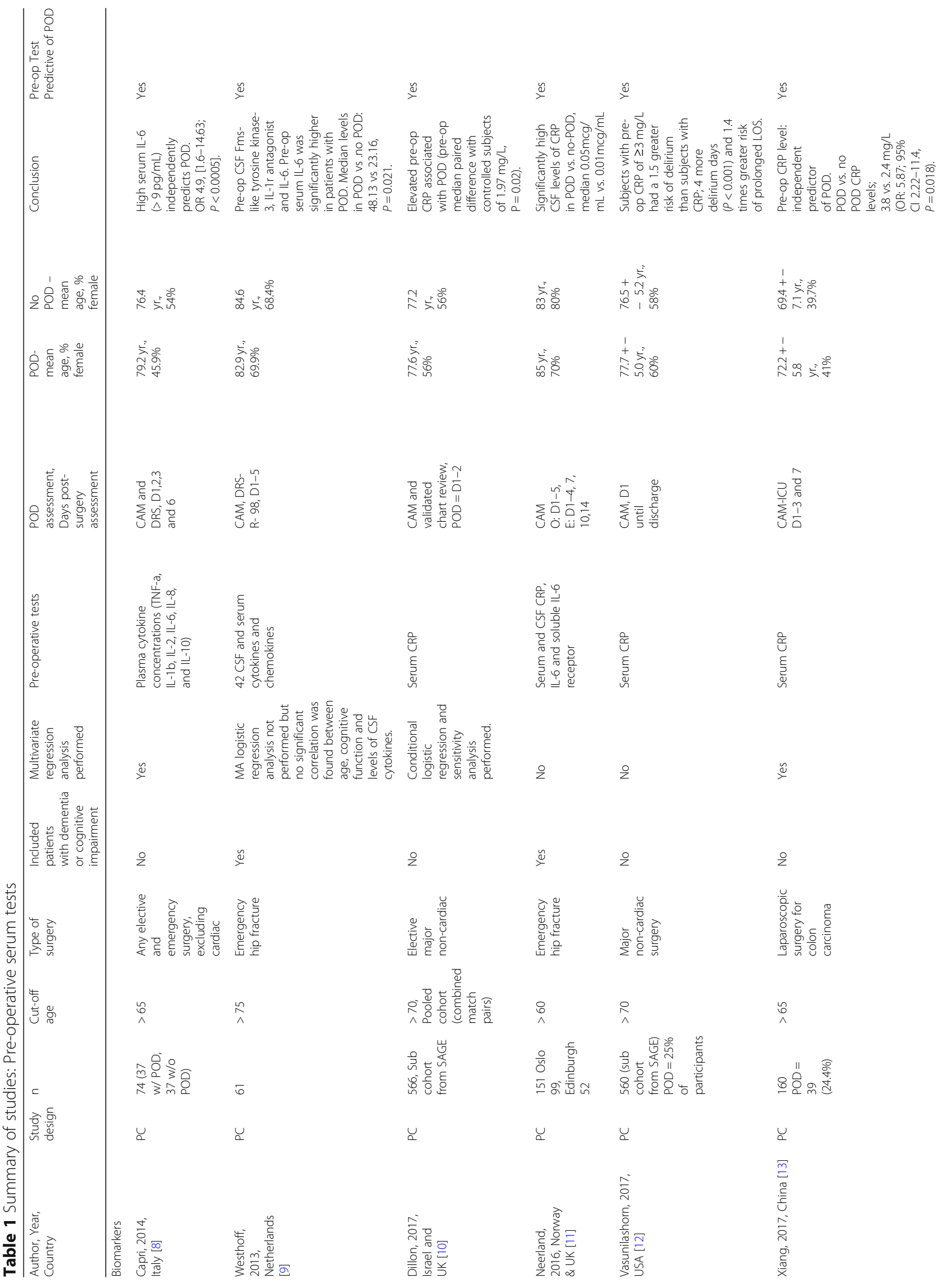




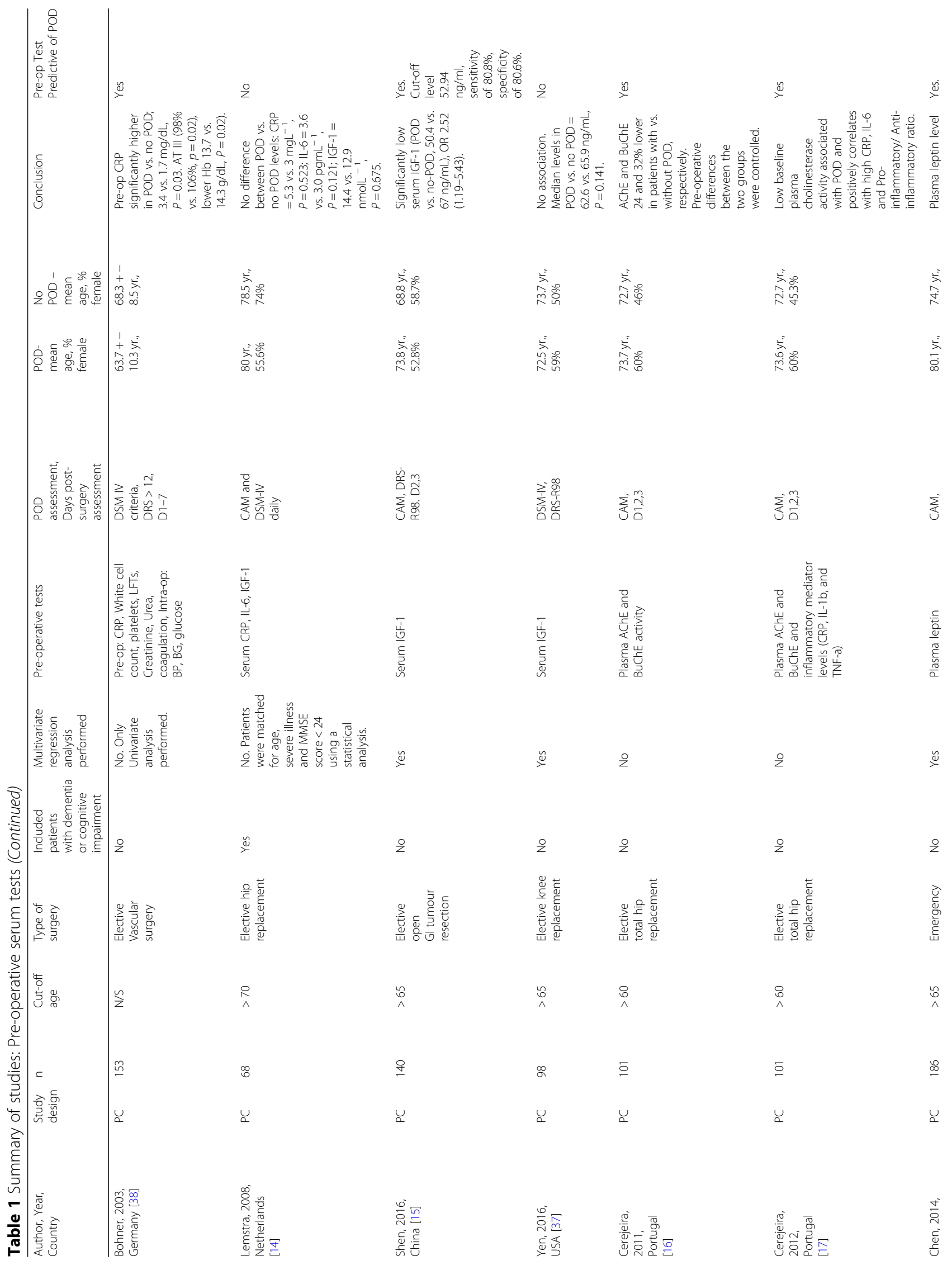




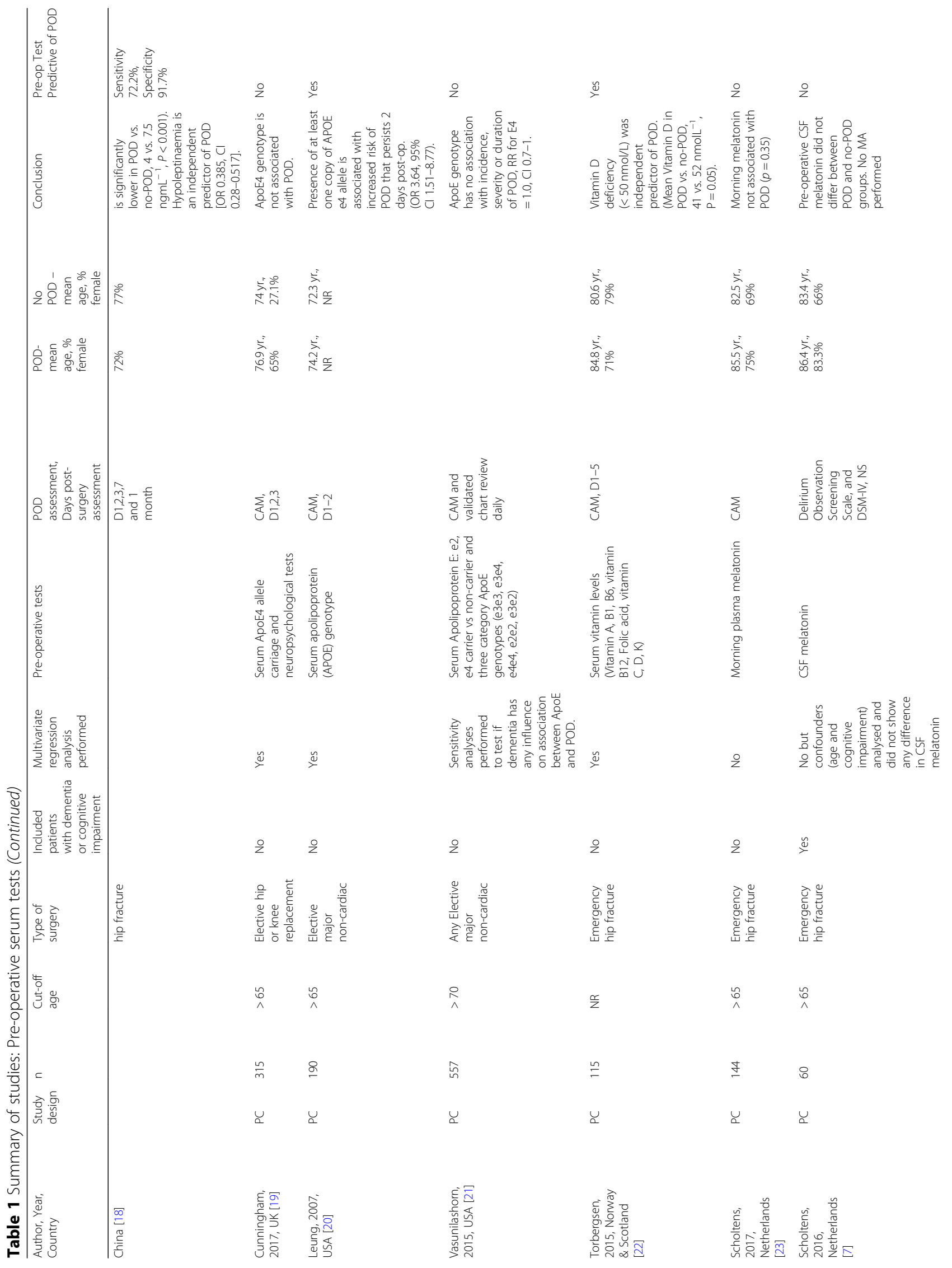




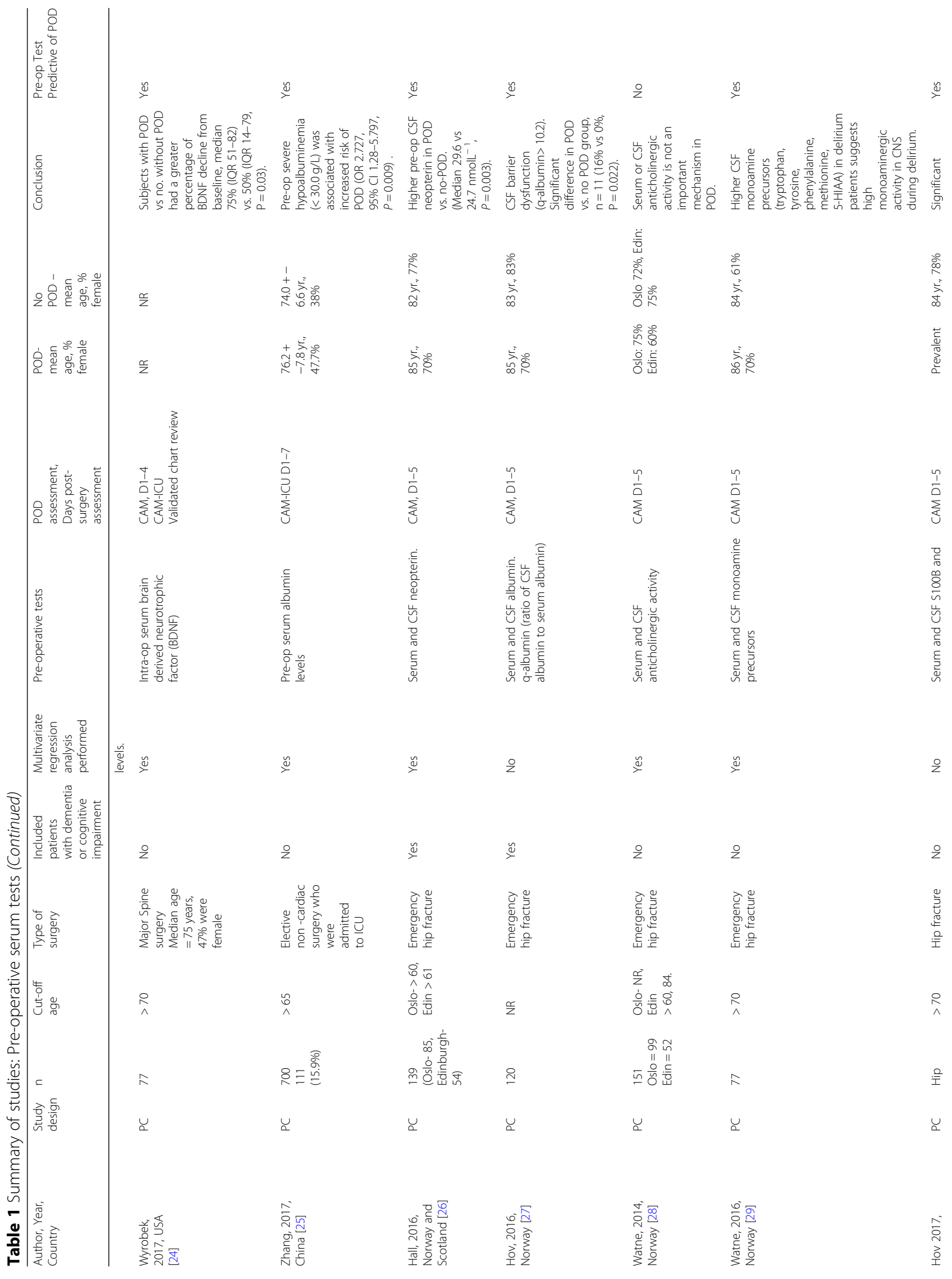


Ayob et al. BMC Anesthesiology

(2019) 19:25

Page 8 of 16

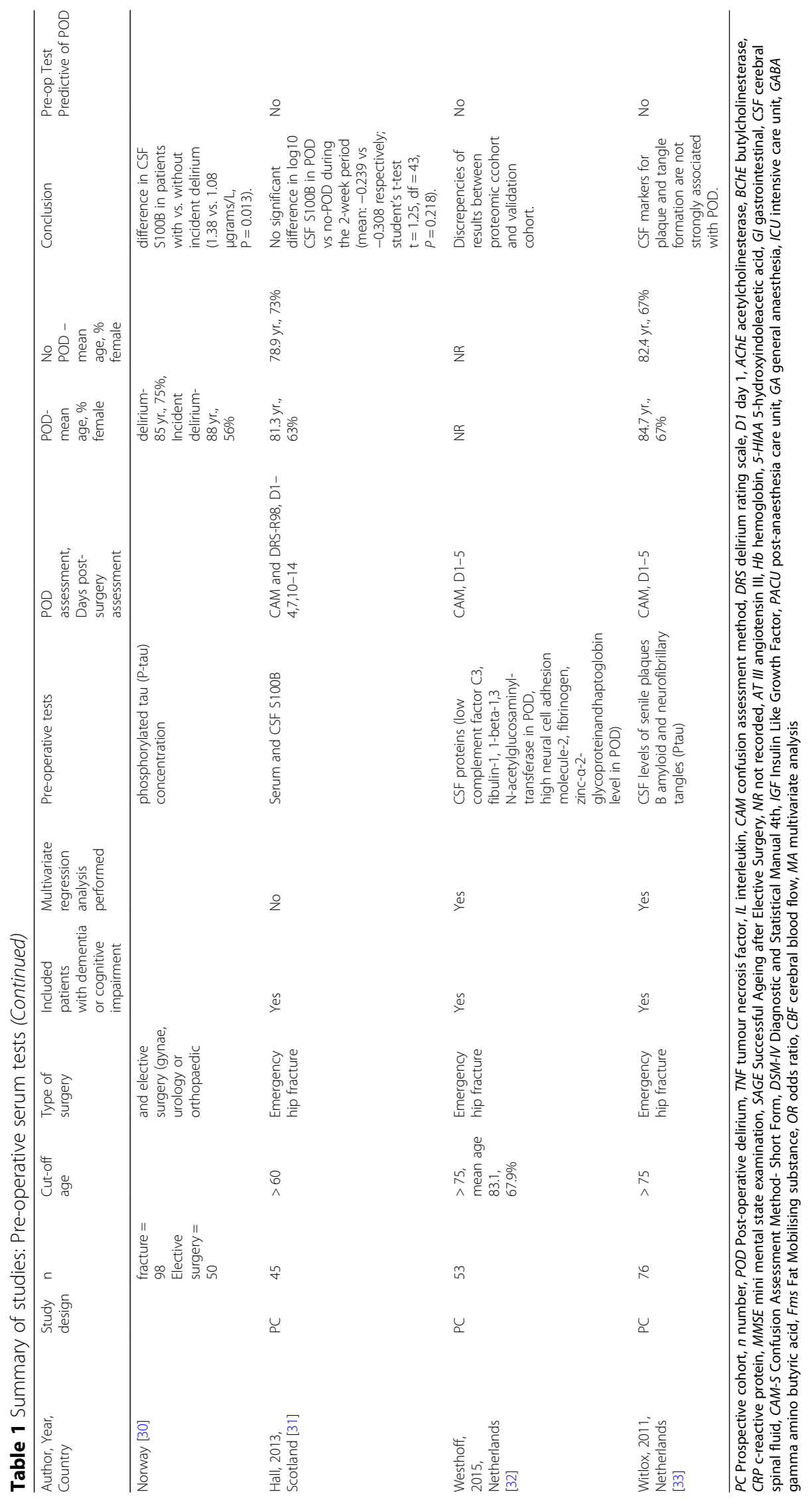




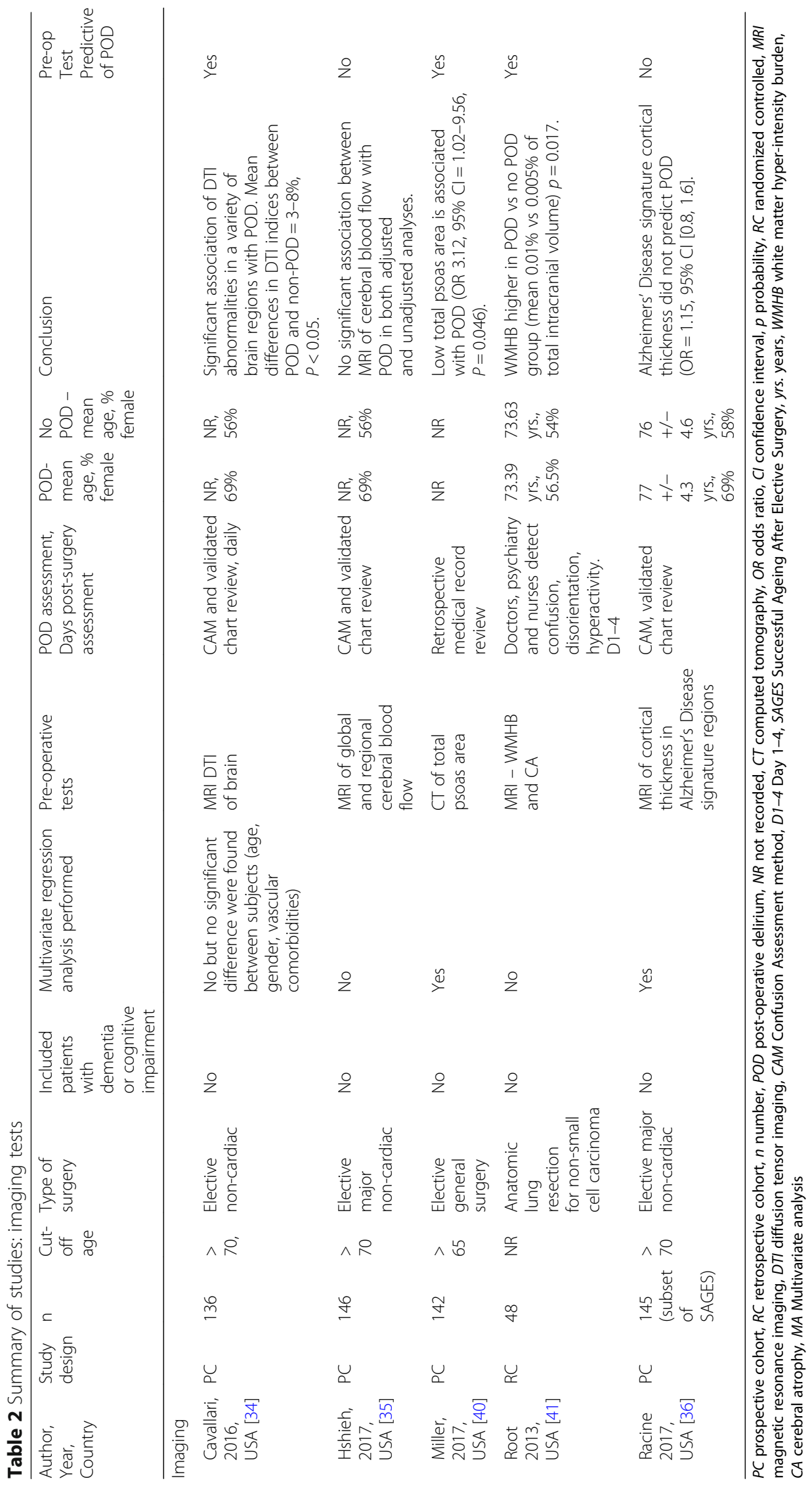


Five studies were major elective orthopaedic surgery $[14,16,17,19,37], 14$ emergency hip fracture surgery $[7, \quad 9,11,18,22,23,26-29,31-33], 10$ major non-cardiac surgery $[8,10,12,20,21,25,30,34-36], 3$ general surgery $[13,15,40], 1$ spine surgery [24], 1 vascular surgery [38] and 1 lung resection surgery [41]. All studies that examined CSF biomarkers involved emergency hip fracture patients. Most studies tested their biomarkers pre-operatively apart from 10 studies which tested their biomarkers in the pre-operative and post-operative period $[10,12,13,17]$.

\section{Risk of Bias}

In general, risk of bias of all studies were low (Table 3 ). For the domain 'study participation', 6/34 (17.6\%) had a moderate risk and 1 study had a high risk of bias. Confounding measurement was found to have moderate risk of bias in $23.5 \%(n=8)$ of the studies. Five studies had moderate bias for attrition measurement and the main reasons were low numbers of eligible participants $[18,31-33]$. Prognosis factor measurement and statistical analysis domains had low bias for all studies due to clear descriptions of valid prognostic factor measurements, and well-chosen statistical models that assessed the data in a non-selective manner respectively.

\section{Biomarkers}

\section{Inflammatory mediators}

Ageing is associated with chronically low levels of inflammation [8]. The association of each biomarker with POD is summarized in Table 4.

\section{II-6}

Two studies showed elevated pre-operative serum IL-6 $[8,9]$, and 1 study did not find any association [14]. One study described their median IL-6 level in the POD group vs. non-POD group was 9 pgmL $^{-1}$ vs. 3.4 pgmL $^{-1}$ [8]. The other study showed no difference in CSF IL-6, however found significant difference in serum levels; (POD vs. non-POD: 48.1 vs. 23.1 pgmL $^{-1}$ ) [9]. The reason for the significant discrepancy in median absolute values between the two studies is not clear. However, the main difference between the two studies which may account for the different IL-6 levels was the use of

Table 3 Summary of risk of bias assessment

\begin{tabular}{llll}
\hline Domains & Low risk n (\%) & Medium risk & High risk \\
\hline Study participation & $27(79.4 \%)$ & $6(17.6 \%)$ & $1(2.9 \%)$ \\
Study attrition & $29(85.3 \%)$ & $5(14.7 \%)$ & $0(0)$ \\
Prognosis factor measurement & $34(100 \%)$ & $0(0)$ & $0(0)$ \\
Outcome measurement & $33(97.1 \%)$ & $0(0)$ & $1(2.9 \%)$ \\
Confounding measurement & $26(76.5 \%)$ & $8(23.5 \%)$ & $0(0)$ \\
Statistical analysis & $34(100 \%)$ & $0(0)$ & $0(0)$ \\
\hline
\end{tabular}

pre-operative anti-inflammatory drugs; whereby the study which excluded its use reported higher IL-6 levels [9], while the study which reported lower IL- 6 levels clearly indicated its main limitation was the absence of information on use of pre-operative anti-inflammatory drugs [8].

\section{CRP}

An association between raised CRP and POD was shown in 5 studies [10-13, 38], and 1 study [14] found no association. Vasinulashorn et al. [12] used a cut-off level for raised CRP as $>3 \mathrm{mgL}^{-1}$; where levels above this showed 1.5 times greater risk of developing delirium. Dillon et al. [10] showed higher CRP levels in POD; measured difference between POD and non-POD groups (median difference of $1.97 \mathrm{mgL}^{-1,} P=0.02$ ). One study in general surgical patients showed higher levels in POD; (POD vs no POD, $3.8 \mathrm{mgL}^{-1}$ vs. $2.4 \mathrm{mgL}^{-1}$ ) [13], as well as another study in vascular patients (POD vs no POD; 3.4 vs. $1.7 \mathrm{mgL}^{-1}$ ) [38]. Neerland et al. [11] compared CSF CRP levels between the two groups; (POD vs. no POD, 0.05 vs. $0.01 \mathrm{mcgmL}^{-1)}$. Based on these studies, a serum level cut-off of CRP $>3 \mathrm{mg} / \mathrm{L}$ is reasonable for predicting POD. The one study which found no association was small and may have been underpowered to show a difference [14]. This study also included patients with baseline cognitive impairment whereas the four studies excluded patients with cognitive impairment.

\section{IGF-1}

IGF-1 is a peptide growth hormone released mainly by the liver. It is neuroprotective; promoting neuronal survival and prevents oxidative stress by inhibiting cytokines. A reduced level may predispose the brain to cytotoxic effects of cytokines [15]. Low serum IGF-1 was significantly associated with POD in 1 study [15], however 2 studies [14, 37] found no association. Only 1 study evaluated the relationship between IGF-1 levels and severity of delirium, and did not find a significant association [37].

\section{Neopterin}

CSF neopterin is a marker of intrathecal immune activation and reflects CNS inflammation. It is synthesized upon stimulation of interferon gamma produced by activated $\mathrm{T}$ cells [42]. Increased neopterin levels were found in patients with delirium, even after adjustment for inflammatory state [43]. Significantly higher levels of pre-operative CSF neopterin were found in the POD group compared to non-POD group (29.6 vs $24.7 \mathrm{nmolL}^{-1}$, $P=0.03)$, even when participants with infection and malignant disease were excluded [26]. 
Table 4 Summary of association of biomarkers and imaging tests with post-operative delirium

\begin{tabular}{|c|c|c|c|c|}
\hline Biomarkers & $\begin{array}{l}\text { Number of studies with positive association } \\
\text { with post-operative delirium }\end{array}$ & References & $\begin{array}{l}\text { Number of studies without an association } \\
\text { with post-operative delirium }\end{array}$ & References \\
\hline IL-6 & 2 & 6,7 & 1 & 12 \\
\hline CRP & 5 & $8,9,10,11,34$ & 1 & 12 \\
\hline |GF-1 & 1 & 13 & 2 & 12,35 \\
\hline Angiontensin III & 1 & 34 & & \\
\hline Hemoglobin & 1 & 34 & & \\
\hline $\begin{array}{l}\text { Reduced Cholinesterase } \\
\text { activity }\end{array}$ & 2 & 14,15 & 0 & \\
\hline $\begin{array}{l}\text { Brain derived neurotrophic } \\
\text { factor (BDNF) }\end{array}$ & 1 & 22 & 0 & \\
\hline Anticholinergic activity & 0 & & 1 & 26 \\
\hline Serum leptin & 1 & 16 & & \\
\hline APO E 4 genotype & 1 & 18 & 2 & 17,19 \\
\hline $\begin{array}{l}\text { Hypovitaminsosis (Vitamin } \\
\text { D deficiency) }\end{array}$ & 1 & 20 & 0 & \\
\hline Melatonin & 0 & & 2 & 5,21 \\
\hline Hypoalbuminemia & 1 & 23 & 0 & \\
\hline Neopterin & 1 & 24 & 0 & \\
\hline Monoamine precursor & 1 & 27 & 0 & \\
\hline CSF Acute phase proteins & 0 & & 1 & 30 \\
\hline S100B & 1 & 28 & 1 & 29 \\
\hline Plaques/tangles & 0 & & 1 & 31 \\
\hline CSF barrier dysfunction & 1 & 25 & 0 & \\
\hline $\begin{array}{l}\text { Magnetic Resonance } \\
\text { Imaging }\end{array}$ & 2 & 32,40 & 2 & 33,41 \\
\hline $\begin{array}{l}\text { Computed Tomography } \\
\text { Imaging }\end{array}$ & 1 & 38 & 0 & \\
\hline
\end{tabular}

IL-6 interleukin-6, CRP C-reactive protein, IGF-1 insulin growth factor-1, APO-E alolipoprotein-E, GABA gamma aminobutyric acid

\section{ApoE4 genotype}

Apolipoprotein E (ApoE) is a component of plasma lipoprotein involved in central acetylcholine synthesis and maintenance of myelin and neuronal membranes during development or after injury. There are three isoforms of ApoE: E2, E3 and E4. The ApoE4 allele is most vulnerable to degradation compared to other isoforms, hence it carries the main genetic risk factor for late onset Alzheimer's disease and is associated with greater risk of amyloid plaque deposition and cerebral amyloid angiopathy [44]. One study reported that the presence of one copy of the e4 allele increased risk of POD that persisted for 2 days post-operatively (28.3\% vs. $11.1 \%$; $P=0.005)$ [20], however two more recent studies reported no association [19, 21]. Interestingly, a recent systematic review of ApoE4 association with delirium concluded no significant association [45].

\section{Anticholinergic activity}

Cholinergic deficiency in the brain is known to play a role in delirium. Acetylcholine degradation not only causes cognitive dysfunction, but also induces inflammation in the central and peripheral nervous systems [46]. Impairment of its receptors might lead to cholinergic deficiency and delirium development [47]. One study investigated serum and CSF anticholinergic activity (AA) using muscarinic radio receptor bioassay, and expressed $\mathrm{AA}$ in terms of atropine equivalents $(\mathrm{pmol} / \mathrm{mL})$. It did not show an association with POD in the two centres in either serum AA in Oslo and in Edinburgh or CSF AA in Oslo and in Edinburgh [28].

\section{Cholinesterases}

Acetylcholinesterase and butylcholinesterases (AChE and $\mathrm{BuChE}$ ) metabolise acetylcholine which results in a lack of cholinergic transmission; a proposed central mechanism of delirium [48]. Cerejeira et al. reported a significantly lower plasma AchE and BuChE of 24 and 32\% respectively in the POD vs. non-POD group [16] and positive correlation between baseline plasma cholinesterase activity and pro-inflammatory/anti-inflammatory ratio $[\mathrm{P} / \mathrm{A}, \mathrm{P} / \mathrm{A}=(\mathrm{IL}$ 
1B + TNF-A + IL-6 + IL-8)/ IL-10] with POD [17]. However, when assessing each mediator individually, no differences were observed between the 2 groups in the latter study [17]. The authors concluded that isolated levels of cytokines in the bloodstream do not necessarily capture the complex homeostatic dysfunction occurring during a delirium episode, and $\mathrm{P} / \mathrm{A}$ ratios might be a more valuable index of cytokine response during a delirium episode [17].

\section{Blood brain barrier dysfunction}

Blood brain barrier dysfunction has been described in dementia [49], but not many studies have described its role in delirium. Pre-operative CSF and serum albumin ratio as a marker of blood brain barrier (BBB) dysfunction ( $\mathrm{q}$ albumin $=$ ratio of CSF albumin to serum albumin, $\mathrm{q}>$ $10=$ CSF barrier dysfunction) has been examined in one study. A significant difference was found in q albumin between the POD and the non-POD group after hip fracture surgery [ $n=11$ ( $16 \%$ vs. $0 \%), P=0.022]$ [27].

\section{Melatonin}

Melatonin is responsible for regulation of circadian rhythm and sleep-wake cycle; the latter being a core feature in delirium. It also inhibits the aggregation of the amyloid beta protein into neurotoxic micro-aggregates responsible for the neurofibrillary tangles in Alzheimer's disease and prevents hyperphosphorylation of the tau protein [50]. Two studies however failed to show an association between pre-operative melatonin levels and POD [10, 23].

\section{Monoamine precursors}

Monoamines such as dopamine, noradrenaline and serotonin have important roles in attention and cognition [51]. The rate of monoamine synthesis in the brain is determined by monoamine precursors (tryptophan, tyrosine and phenylalanine). One study examined the association between POD and amino acids and/or monoamine metabolites (5-hydroxyindoleacetic acid; 5-HIAA) levels in the CSF in patients with and without pre-existing dementia. Significantly higher CSF levels of tryptophan, 0.8 vs. $1.1 \mu \mathrm{molL}^{-1}, P=0.042$; tyrosine, 5 vs. $8 \mu \mathrm{molL}^{-1}, P=0.028$; phenylalanine, 9 vs. $14 \mu \mathrm{molL}^{-1}$, $P=0.014$; methionine, 3 vs. $4 \mu \mathrm{molL}^{-1}, P=0.03$ and monoamine metabolite 5 -HIAA, 138 vs. $170 \mathrm{nmolL}^{-1}$, $P=0.048$ were found in the POD vs. the non-POD group after hip fracture surgery [29]. These findings were found in patients without pre-existing dementia. When patients with and without dementia were analysed together, only CSF methionine and serum taurine were significantly different between the POD and non-POD group. The study suggested that increased monoaminergic activity is associated with delirium in hip fracture patients.

\section{CSF protein}

Acute phase proteins (complement c3, fibrinogen and haptoglobin) may be associated with the inflammatory process in delirium [52]. Complement factor C3, contactin1, fibulin-1 and I-beta-1,3-N-acetylglucosaminyl transferase were significantly lower, while neural cell adhesion molecule-2, fibrinogen, zinc- $\alpha$-2-glycoprotein and haptoglobin levels were significantly higher in the POD vs. control group in their proteomic cohort after hip fracture surgery, but failed to demonstrate this in their validation cohort [32]. The authors concluded that their inconsistent results were due to minor differences between the two cohorts (patients in the validation group were older than the proteomic group) and also due the complexity of the pathophysiology of delirium.

\section{CSF S100B}

$\mathrm{S} 100 \mathrm{~B}$ is a calcium binding protein in the CNS mainly secreted by astrocytes under metabolic stress. Elevated levels have been implicated as a marker of acute brain damage [53]. One study in both hip fracture surgery and the elective surgery showed a significant difference (POD vs. no

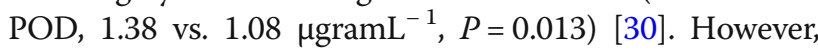
this difference was confined to patients with pathological concentrations of phosphorylated-tau (>60 ng/L) - an abnormally phosphorylated protein; its presence in the CSF reflects early signs of Alzheimer's dementia [54]. Another study however found no significant difference in S100B concentration between POD and non-POD groups having hip fracture surgery [31]. The reason for inconsistencies were not clear, but the populations in both studies differed; with a higher proportion of nursing home and dementia patients in the positive study [30].

\section{Neurofibrillary plaques}

Senile plaques composed of beta-amyloid (AB1-42) and neurofibrillary tangles (phosphorylated tau) are commonly associated with the pathological process in Alzheimer's dementia [51]. The relationship of Alzheimer's disease and susceptibility of POD is not well understood, however, in one study, CSF markers for plaques and tangles were not significantly associated with POD risk in elderly hip fracture patients [33].

\section{Leptin}

Leptin modulates immune response, and hypoleptinaemia can increase the production of pro-inflammatory cytokines that could result in cognitive impairment and delirium [55]. Low plasma leptin was associated with POD after femoral neck fracture surgery in one study (POD vs. no POD: 4.6 vs 7.5 ngmL $^{-1}, p<0.001$ ) [18]. 


\section{Hypovitaminosis}

Vitamin D is a steroid hormone, which acts on neuroanatomic areas that sub-serve cognition. Hypovitaminosis and its link with cognitive impairment has been described in a few studies $[56,57]$. Theoretically, Vitamin D may have a role in protecting the brain from delirium by preventing oxidative stress in hippocampal neurons [58] and promotes neuroprotection by modulating the production of choline acetyltransferase [59]. One study examining serum vitamin levels in hip fracture patients showed that Vitamin D deficiency $\left(<50 \mathrm{nmolL}^{-1}\right)$ was an independent predictor of POD; mean Vitamin D levels in POD vs. no POD: 41 vs. $52 \mathrm{nmolL}^{-1}, p=0.05$ [22].

\section{Hypoalbuminemia}

Hypoalbuminemia is used as a marker of malnutrition and several studies have shown hypoalbuminemia as a risk factor for POD [60, 61]. Severe hypoalbuminaemia $(<30 \mathrm{~g} / \mathrm{L})$ but not mild $(30.1-35 \mathrm{~g} / \mathrm{L})$ or moderate $(35.1-40 \mathrm{~g} / \mathrm{L})$ hypoalbuminemia was significantly associated with a twofold higher risk of POD [25].

\section{Brain derived neurotrophic factor (BDNF)}

BDNF is a protein that influences neuroplasticity and neurotransmission, learning, memory and cognition. One study showed that greater decline in BDNF levels from baseline was associated with an increased incidence of delirium in patients undergoing spine surgery; POD vs no POD $75 \%$ vs $50 \%, p=0.03$ [24].

\section{Angiotensin III (AT III) and haemoglobin}

One study found that a lower AT III level (POD vs no POD, $98 \%$ vs. $106 \%, p=0.02$ ); and haemoglobin level pre-operatively (POD vs no POD, 13.7 vs. 14.3 $\mathrm{g} / \mathrm{dL}, P=0.02)$ increase the risk of developing POD in vascular surgery [38].

\section{Imaging}

Integration of imaging results with information such as structural integrity or changes, vascular profile, or neurotransmitter imbalances can give rise to computational modelling of POD.

\section{Magnetic resonance imaging (MRI)}

Various techniques in MRI have been reported in predicting POD. Diffusion tensor imaging is a MRI technique that shows microstructural integrity of the brain parenchyma and has been applied to map underlying functional impairments. A significant association of premorbid diffusion tensor imaging abnormalities with delirium incidence and severity in a variety of brain regions, particularly the cerebellum was shown in one study [34]. In a cohort of patients undergoing lung resection surgery, cerebral MRI showed higher proportion of white matter hyper-intensities in the POD vs. non-POD group, (mean $0.01 \%$ vs $0.005 \%$ of total intracranial volume, $P=0.017$ ) [41]. However, two studies involving MRI; one study on global and regional cerebral blood flow [35], and another study of cortical thickness in Alzheimer's disease signature region [36] showed no association with POD.

\section{Computed tomography imaging (CT)}

One study found that low total psoas area (TPA), a marker of surgical frailty was associated with an almost 3-fold increased risk of POD [40].

The current evidence on pre-operative biomarkers associated with POD are summarised in Table 4.

\section{Discussion}

Biomarkers can be useful to improve understanding of pathophysiology of POD, develop new treatment for POD, predict possible long term effects of delirium, stratify high risk patients and allow informed decision making for patients about their risks for surgery. Many biomarkers associated with dementia or hypothesized causes of post-operative delirium have been investigated.

In this review, CRP was the most promising biomarker for predicting POD. Although there is variability in CRP measurement and cut-off levels in each study, the slightly raised CRP levels that were predictive in the studies suggests that there may be an association of heightened innate inflammatory response leading to POD. There is growing evidence of raised innate inflammation associated with POD as evidenced by elevation of pre-operative inflammatory markers such as CRP, IL-6 and neopterin. One hypothesis suggests an exaggerated activation of the innate immune system results in functional changes in neurons which may lead to cognitive and behavioural changes [62]. The degree of neuro-inflammation tends to increase with age, which may explain why elderly individuals are more prone to developing POD when administered anaesthesia [63]. Predisposed individuals who experience stressors such as during infection or surgery may also exhibit crossing of systemic inflammatory mediators across the blood-brain barrier and activation of brain microglia, which eventually leads to neuro-inflammation and delirium $[12,64]$.

CRP is a non-specific acute phase marker in inflammation, infection and tissue damage. Although raised CRP may be non-specific to POD, the initial findings are encouraging for further research. Other biomarkers that were found to be associated with POD include BDNF, reduced cholinesterase activity, serum leptin, hypoalbuminaemia and vitamin D deficiency. However, we cannot draw any conclusions as to their roles in POD due to the limited number of studies. 
The results for CSF biomarkers and imaging as predictors for POD are inconclusive. The studies that examined CSF biomarkers were for patients undergoing spinal anaesthesia, thus the patients did not require an additional lumbar puncture to obtain CSF. The risks and invasiveness of obtaining CSF (if a patient was not already undergoing spinal anaesthesia) and costs applied to imaging may limit their practicality in clinical practice.

\section{Strengths and limitations}

The strengths of this review include the comprehensive literature search using different search terms from several large databases and the total number of patients included from geographically diverse areas.

The limitations of the review are that most studies were relatively small and some biomarkers were only studied in one study, hence limiting quantitative assessment and meta-analysis of results. Some studies used the same cohort of patients to test different biomarkers. Secondly, we restricted our search only to English language articles. Most studies were conducted in orthopaedic surgery; therefore, the results may not be generalised to other types of surgery. Another limitation is that some studies included patients with pre-existing cognitive impairment or dementia [7, 9, 11, 14, 26, 27, 31-33]. However, no statistical difference were found in pre-operative cognitive scores between control and POD groups in all the studies. Some studies did not perform multivariate analysis which may not account fully for potential confounders $[7,9,11$, $12,14,16,17,21,23,27,30-32,34,41]$. The timing of POD assessment also varied, and no study mentioned if they included PACU delirium. However, whether delirium in PACU is considered POD is subject to debate, and although its assessment is different (CAM-ICU has a higher specificity than sensitivity for delirium when used in PACU) [2], the relatively high incidence of PACU delirium (16.4\%) [3] may be relevant in accounting for the accurate incidence of POD.

Therefore, further research should incorporate a variety of surgical procedures, align timing of POD assessment including PACU delirium, using multivariate models and adjust for confounding factors.

\section{Conclusions}

The current literature regarding pre-operative biochemical markers and imaging tests for predicting post-operative delirium is still in its infancy. There is some evidence that serum CRP levels $>3 \mathrm{mgL}^{-1}$ may predict a high risk of developing POD, and incorporating it along with other patient and surgical predisposing factors of POD may increase the identification of high risk patients. However, this should be interpreted with caution due to heterogeneity of predictive test values and the small sample size of the studies precluded sensitivity analysis to demonstrate significant association. On the other hand, delineating specific biomarkers may also lead to forming composite markers (combining 2 or more biomarkers) which could increase predictive value. From a clinical perspective, utilising predictive biomarkers may allow better clinical decision making and management of high risk patients to specific recovery units.

\section{Additional files}

Additional file 1: Search strategy. (DOCX $129 \mathrm{~kb})$

Additional file 2: Supplementary Table S1. QUIPS Risk of bias assessment.. (DOCX $88 \mathrm{~kb}$ )

\section{Abbreviations}

QoL: Quality of life; CAM-ICU: Confusion assessment methd-intensive care unit; 5-HIAA: 5-hydroxyindoleacetic acid; 6 RCT: Randomised control trial;

AB1-42: Amyloid-b 1-42; AChE: Acetylcholinesterase; ApoE: Alolipoprotein-E; AT III: Angiotensin-III; BBB: Blood brain barrier; BDNF: Brain derived neurotrophic factor; BuChE: Butylcholinesterase; CAM: Confusion Assessment Method; CRP: CReactive protein; CSF: Cerebral spinal fluid; CT: Computed tomography; DRS: Delirium rating scale; DSM: Diagnostic and statistical manual for mental disorders; IGF-1: Insulin-like growth factor-1; IL-10: Interleukin-10; IL-

1B: Interleukin-1B; IL-6: Interleukin-6; IL-8: Interleukin-8; MRI: Magnetic resonance 13 imaging; OR: Odds ratio; P/A: Pro-inflammatory/anti-inflammatory ratio; POD: Post-operative delirium; PRISMA: Preffered reporting items for systematic review; QUIPS: Quality in Prognostic Studies; SAGE: Successful ageing after elective surgery; TNF-A: Tumour necrosis factor-Alpha; TPA: Total psoas area

\section{Acknowledgements}

The authors would like to thank Marina Englesakis for her assistance with performing the literature search of relevant articles.

\section{Funding}

Department of Anesthesia, Toronto Western Hospital, University Health Network. The department had no role in the design of the study and collection, analysis, interpretation.

of data and in writing the manuscript.

\section{Availability of data and materials}

All databases are included in the section of Methods and Materials.

\section{Authors' contributions}

FA extracted data from included studies, interpreted data, prepared the tables, wrote and edited the manuscript. EL and $\mathrm{GH}$ performed the literature search, extracted relevant studies, data analysis, prepared the tables and figures, reviewed and helped write the manuscript. HEB helped write and edit the manuscript. FC helped interpret data, helped write and edit the manuscript. JW coordinated the study, designed the study, interpreted data, helped write and edit the manuscript. All authors have read and approved the manuscript.

Ethics approval and consent to participate

Ethics approval and consent to participate are not needed for systematic reviews.

Consent for publication

Not applicable.

\section{Competing interests}

JW reports grants from the Ontario Ministry of Health and Long-Term Care, Anesthesia Patient Safety Foundation and Acacia Pharma outside of the submitted work. FC reports research support from the Ontario Ministry of Health and Long-Term Care, University Health Network Foundation, ResMed Foundation, Acacia Pharma, Medtronic grants to institution, Up-to-date royalties, STOP-Bang propriety to University Health Network outside of the submitted work. The other authors have no conflicts of interest to report. 


\section{Publisher's Note}

Springer Nature remains neutral with regard to jurisdictional claims in published maps and institutional affiliations.

\section{Author details \\ 'Department of Anesthesia, Toronto Western Hospital, University Health Network, 2-434 McLaughlin Wing, 399 Bathurst Street, Toronto, ON M5T 2S8, Canada. ${ }^{2}$ Faculty of Medicine, University of Toronto, 1 King's College Circle, Medical Sciences Building, Room 2109, Toronto, ON M5S 1A8, Canada. ${ }^{3}$ Department of Anesthesia, Trillium Health Partners, Mississauga Hospital, 100 Queensway, West, Mississauga, ON L5B 1B, Canada. 'Women's College Hospital, Toronto, Ontario, 76 Grenville St, Toronto, ON M5S 1B2, Canada.}

Received: 31 July 2018 Accepted: 8 February 2019

Published online: 23 February 2019

\section{References}

1. Deiner $\mathrm{S}$, Silverstein $\mathrm{JH}$. Postoperative delirium and cognitive dysfunction. $\mathrm{Br}$ J Anaesth. 2009;103:41-6.

2. Card E, Pandharipande P, Tomes C, Lee C, Wood J, Nelson D, et al. Emergence from general anaesthesia and evolution of delirium signs in the post-anaesthesia care unit. Br J Anaesth. 2015;115(3):411-7.

3. Hernandez BA, Lindroth H, Rowley P, Boncyk C, Raz A, Gaskell A, et al. Post-anaesthesia care unit delirium: incidence, risk factors and associated adverse outcomes. Br J Anaesth. 2017;119(2):288-90.

4. Mashour GA, Woodrum DT, Avidan MS. Neurological complications of surgery and anaesthesia. Br J Anaesth. 2015;114(2):194-203.

5. Lee $\mathrm{A}$, JL M, Joynt GM, Chiu CH, Lai VKW, et al. Risk prediction models for delirium in the intensive care unit after cardiac surgery: a systematic review and independent external validation. Br J Anaesth. 2017;118(3):391-9.

6. Hayden JA, Van der Windt DA, Cartwright $J$, Cote P, Bombardier C. Assessing bias in studies of prognostic factors. Ann Intern Med. 2013; 158(4):280-6.

7. Scholtens RM, van Munster BC, van Faassen M, van Kempen MF, Kema IP, de Rooji SE. Preoperative CSF melatonin concentrations and the occurrence of delirium in older hip fracture patients: a preliminary study. PLoS One. 2016;11(12):e0167621.

8. Capri M, Yani SL, Chattat R, Fortuna D, Bucci L, Lazarini C, et al. Pre-operative high-IL-6 blood level is a risk factor of post-operative delirium onset in old patients. Front Endocrinol. 2014;5:173.

9. Westhoff D, Witlox J, Koenderman L, Kalisvaart KJ, de Jonghe J, Stijn MF, et al. Preoperative cerebrospinal fluid cytokine levels and the risk of postoperative delirium in elderly hip fracture patients. J Neuroinflam. 2013; 10(122):0-10.

10. Dillon ST, Vasunilashorn SM, Ngo L, Otu H, Inouye S, Jones RN, et al. Higher C-reactive protein levels predict postoperative delirium in older patients undergoing major elective surgery: a longitudinal nested case-control study. Biol Psychiatry. 2017;81(2):145-53.

11. Neerland BE, Hall RJ, Seljeflot I, Frihagen F, MacLullich A, Raeder J, et al. Associations between delirium and preoperative cerebrospinal fluid C-reactive protein, Interleukin-6, and Interleukin-6 receptor in individuals with acute hip fracture. J Am Geriatr Soc. 2016:64(7):1456-63.

12. Vasunilashorn SM, Dillon ST, Inouye SK, Ngo L, Fong T, Jones R, et al. High C-reactive protein predicts delirium incidence, duration, and feature severity after major noncardiac surgery. J Am Geriatr Soc. 2017;65(8):e109-16.

13. Xiang $\mathrm{D}$, Xing $\mathrm{H}$, Tai $\mathrm{H}$, Xie $\mathrm{G}$. Preoperative $\mathrm{C}$-reactive protein as a risk factor for postoperative delirium in elderly patients undergoing laparoscopic surgery for Colon carcinoma. Biomed Res Int. 2017;2017:5635640.

14. Lemstra AW, Kalisvaart KJ, Vreeswijk R, van Gool WA, Eikelenboom P. Pre-operative inflammatory markers and the risk of postoperative delirium in elderly patients. Int J Geriatr Psychiatry. 2008;23(9):943-8.

15. Shen H, Shao Y, Chen J, Guo J. Insulin-like growth Factor-1, a potential predicative biomarker for postoperative delirium among elderly patients with open abdominal surgery. Curr Pharm Des. 2016;22:5879-83.

16. Cerejeira J, Batista P, Noguira V, Firmino H, Vaz-Serra A, Mukaetova-Ladinska EB. Low preoperative plasma cholinesterase activity as a risk marker of postoperative delirium in elderly patients. Age Ageing. 2011;40(5):621-6.

17. Cerejeira J, Nogueira V, Luis P, Vaz-Serra A, Mukaetova-Ladinska E. The cholinergic system and inflammation: common pathways in delirium pathophysiology. J Am Geriatr Soc. 2012;60(4):669-75.
18. Chen XW, Shi JW, Yang PS, Wu ZQ. Preoperative plasma leptin levels predict delirium in elderly patients after hip fracture surgery. Peptides. 2014:57:31-5.

19. Cunningham EL, Mawhinney T, Beverland D, O'Brien S, McAuley D, Cairns $R$, et al. Observational cohort study examining apolipoprotein E status and preoperative neuropsychological performance as predictors of post-operative delirium in an older elective arthroplasty population. Age Ageing. 2017;46(5):779-86.

20. Leung JS, Wand L, Poon Y, Kwok A, Kane P, Pullinger CR. Apolipoprotein E e4 allele increases the risk of early post-operative delirium in older patients undergoing non-cardiac surgery. Anesthesiology. 2007;107:406-11.

21. Vasunilashorn S, Ngo L, Kosar CM, Fong TG. Does apolipoprotein E genotype increase risk of postoperative delirium? Am J Geriatr Psych. 2015; 23(10):1029-37.

22. Torbergsen AC, Watne LO, Frihagen F, Wylier TB, Brugaard A, Mowe M. Vitamin deficiency as a risk factor for delirium. Eur Geriatric Med. 2015;6(4):314-8.

23. Scholtens RM, van Munster BC, van Faasen M, van Kempen MF, Kema IP, de Rooji SE. Plasma melatonin levels in hip fracture patients with and without delirium: a confirmation study. Mech Ageing Dev. 2017;167:1-4.

24. Wyrobek J, LaFlam A, Max L, Tian J, Neufeld KJ, Kebaish KM, et al. Association of intraoperative changes in brain-derived neurotrophic factor and postoperative delirium in older adults. Br J Anaesth. 2017;119(2):324-32.

25. Zhang DF, Su X, Meng ZT, Cui F, Li HL, Wang DX, et al. Preoperative severe hypoalbuminemia is associated with an increased risk of postoperative delirium in elderly patients: results of a secondary analysis. J Crit Care. 2017:44:45-50.

26. Hall RJ, Watne LO, Idland AV, Raeder J, Frihagen F, MacLullich A, et al. Cerebrospinal fluid levels of neopterin are elevated in delirium after hip fracture. J Neuroinflam. 2016:13(1):170.

27. Hov KR, Berg JP, Frihagen F, Raedr J, Hall R, Wyller T, et al. Blood-cerebrospinal fluid barrier integrity in delirium determined by Q-albumin. Dement Geriatr Cogn Disord. 2016;41(3-4):192-8.

28. Watne LO, Hall RJ, Molden E, Raeder J, Frihagen F, MacLullich A, et al. Anticholinergic activity in cerebrospinal fluid and serum in individuals with hip fracture with and without delirium. J Am Geriatr Soc. 2014;62(1):94-102.

29. Watne LO, Idland AV, Fekkes D, Raeder J, Frihagen F, Ranhoff AH, et al. Increased CSF levels of aromatic amino acids in hip fracture patients with delirium suggests higher monoaminergic activity. BMC Geriatr. 2016;16:149.

30. Hov KR, Bolstad N, Idland AV, Zetterberg H, Blennow K, Chaudury FK, et al. Cerebrospinal fluid S100B and Alzheimer's disease biomarkers in hip fracture patients with delirium. Dement Geriatr Cogn Dis Extra. 2017;7(3):374-85.

31. Hall RJ, Ferguson K, Andrews M, Green A, White T, Armstrong I, et al. Delirium and cerebrospinal fluid S100B in hip fracture patients: a preliminary study. Am J Geriatr Psych. 2013;21(12):1239-43.

32. Westhoff D, Witlox J, van Aalst C, de Rooji S, van Munster D, et al. preoperative protein profiles in cerebrospinal fluid in elderly hip fracture patients at risk for delirium: a proteomics and validation study. BBA Clin. 2015:4:115-22.

33. Witlox J, Kalisvaart KJ, de Jonghe JF, Verwey NA, Stijn M, Houdijk A, et al. Cerebrospinal fluid beta-amyloid and tau are not associated with risk of delirium: a prospective cohort study in older adults with hip fracture. J Am Geriatr Soc. 2011:59(7):1260-7.

34. Cavallari M, Dai W, Guttmann CR, Meier DS, Ngo LH, Hshieh TT, et al. Neural substrates of vulnerability to postsurgical delirium as revealed by presurgical diffusion MRI. Brain. 2016;139(4):1282-94.

35. Hshieh T, Dai W, Cavallari M, Guttmann C, Meier DS, Schmitt E, et al. Cerebral blood flow MRI in the nondemented elderly is not predictive of post-operative delirium but is correlated with cognitive performance. J Cereb Blood Flow Metab. 2017;37(4):1386-97.

36. Racine AM, Fong TG, Travison TG, Jones R, Gou Y, Vasunilashorn $S$, et al. Alzheimer's related cortical atrophy is associated with postoperative delirium severity in persons without dementia. Neurobiol Aging. 2017; 59:55-63.

37. Yen TE, Allen JC, Rivelli SK, Patterson SC, Metcalf MR, Flink BJ, et al. Association between serum IGF-I levels and postoperative delirium in elderly subjects undergoing elective knee arthroplasty. Sci Rep. 2016;6:20736.

38. Bohner H, Hummel TC, Habel U, Miller C, Reinbott S, Yang Q, et al. Predicting delirium after vascular surgery: a model based on pre- and intraoperative data. Ann Surg. 2003:238(1):149-56.

39. Schmitt EM, Sacynski JS, Kosar CM, Jones RM, Alsop DC, Fong TG, et al. The successful aging after elective surgery (SAGES) study: cohort description and data quality procedures. J Am Geriatr Soc. 2015;63(12):2463-71. 
40. Miller AL, Englesbe MJ, Diehl KM, Chan CL, Cron DC, Derstine BA, et al. Preoperative Psoas Muscle Size Predicts Post-operative Delirium in Older Adults Undergoing surgery: A pilot cohort study. Am Geriatr Soc. 2017;65(1):23-4.

41. Root JC, Pryor KO, Downey R, Alici Y, Davis M, Holodny A, et al. Association of pre-operative brain pathology with postoperative delirium in a cohort of non-small cell lung cancer patients undergoing surgical resection. Psycho-Oncology. 2013;22(9):2087-20.

42. Eisenhut M. Neopterin in diagnosis and monitoring of infectious diseases. J Biomark. 2013;2013:196432.

43. Egberts A, Wijnbeld EH, Fekkes D, van der Ploeg MA, Ziere G, Hooijkaas H, et al. Neopterin: a potential biomarker for delirium in elderly patients. Dement Geriatr Cogn Disord. 2015;39(1-2):116-24.

44. Caplan GA, Tai J, Mohd Hanizan F, McVeigh CL, Hill MA, Poljak A. Cerebrospinal Fluid Apolipoprotein E Levels in Delirium. Dement Geriatr Cogn Disord Extra. 2017:7(2):240-8.

45. Adamis D, Meagher D, Williams J, Mulligan O. McCarthy G. A systematic review and meta-analysis of the association between the apolipoprotein $\mathrm{E}$ genotype and delirium. Psychiatr Genet. 2016;26(2):53-9.

46. Kitajima Y, Hori K, Konishi K, Tani M, Tomioka H, Akashi N, et al. A review of the role of anticholinergic activity in Lewy body disease and delirium. Neurodegener Dis. 2015;15(3):162-7.

47. Plaschke K, Petersen KA, Frankenhauser S, Weigand MA, Kopitz J, Bardenheuer HJ. The impact of plasma cholinergic enzyme activity and other risk factors for the development of delirium in patients receiving palliative care. J Pain Symptom Manag. 2016;52(4):525-32.

48. Tampi RR, Tampi DJ, Ghori AK. Acetylcholinesterase inhibitors for delirium in older adults. Am J Alzheimers Dis Other Demen. 2016;31(4):305-10.

49. Zeevi N, Pachter J, McCollohg LD, Wolfson KGA. The blood-brain barrier: geriatric relevance of a critical brain-body interface. J Am Geriatr Soc. 2010;58(9):1749-57.

50. Sultan SS. Assessment of role of perioperative melatonin in prevention and treatment of postoperative delirium after hip arthroplasty under spinal anesthesia in the elderly. Saudi J Anaesth. 2010:4(3):169-73.

51. Maldonado JR. Neuropathogenesis of delirium: review of current etiologic theories and common pathways. Am J Geriatr Psychiatry. 2013;21(12):1190-222.

52. Poljak A, Hill M, Hall RJ, MacLullich AM, Raftery MJ, Tai J, et al. Quantitative proteomics of delirium cerebrospinal fluid. Transl Psychiatry. 2014;4:e477.

53. Michetti F, Corvino V, Geloso MC, Lattanzi W, Bernardini C, Serpero L, et al. The S100B protein in biological fluids: more than a lifelong biomarker of brain distress. J Neurochem. 2012;120(5):644-59.

54. Kandimalla RJ, Prabhakar S, Wani WY, Kaushal A, Gupta N, Sharma DR, et al. CSF p-tau levels in the prediction of Alzheimer's disease. Biol Open. 2013;2(11):1119-24.

55. Zeki Al Hazzouri A, Stone KL, Haan MN, Yaffe K. Leptin, mild cognitive impairment, and dementia among elderly women. J Gerontol A Biol Sci Med Sci. 2013;68(2):175-80.

56. Llewellyn DJ, Langa KM, Lang IA. Serum 25-hydroxyvitamin D concentration and cognitive impairment. J Geriatr Psychiatry Neurol. 2009;22(3):188-95.

57. Annweiler C, Allali G, Allain P, Bridenbaugh S, Schott AM, Kressig RW, et al. Vitamin $D$ and cognitive performance in adults: a systematic review. Eur J Neurol. 2009;16(10):1083-9.

58. Ibi M, Sawada H, Nakanishi M, Kume T, Katsuki H, Kaneko S, et al. Protective effects of 1 alpha,25-(OH)(2)D(3) against the neurotoxicity of glutamate and reactive oxygen species in mesencephalic culture. Neuropharmacology. 2001;40(6):761-71.

59. Sonnenberg J, Luine VN, Krey LC, Christakos S. 1,25-Dihydroxyvitamin D3 treatment results in increased choline acetyltransferase activity in specific brain nuclei. Endocrinology. 1986;118(4):1433-9.

60. Guo Y, Jia P, Zhang J, Wang $X$, Jiang $H$, Jiang W. Prevalence and risk factors of postoperative delirium in elderly hip fracture patients. J Int Med Res. 2016:44(2):317-27.

61. Patti R, Saitta M, Cusumano G, Termine G, Di Vita G. Risk factors for postoperative delirium after colorectal surgery for carcinoma. Eur J Oncol Nurs. 2011;15(5):519-23.

62. Maclullich AM, Ferguson KJ, Miller T, de Rooji SE, Cunningham C. Unravelling the pathophysiology of delirium: a focus on the role of aberrant stress responses. J Psychosom Res. 2008;65(3):229-38.

63. Hala M. Pathophysiology of postoperative delirium: systemic inflammation as a response to surgical trauma causes diffuse microcirculatory impairment. Med Hypotheses. 2007;1:194-6.

64. Inouye SK, Westendorp RG, Saczynski JS. Delirium in elderly people. Lancet. 2014;383(9920):911-22.

Ready to submit your research? Choose BMC and benefit from:

- fast, convenient online submission

- thorough peer review by experienced researchers in your field

- rapid publication on acceptance

- support for research data, including large and complex data types

- gold Open Access which fosters wider collaboration and increased citations

- maximum visibility for your research: over $100 \mathrm{M}$ website views per year

At $\mathrm{BMC}$, research is always in progress.

Learn more biomedcentral.com/submissions 